'Escuela de Salud Pública, Facultad de Medicina, Universidad de Chile. ${ }^{2}$ Departamento de Psiquiatría y Salud Mental Sur, Universidad de Chile. ${ }^{3}$ Departamento Médico, Superintendencia de Seguridad Social.

Declaración de conflicto de intereses: Este trabajo fue financiado por la Superintendencia de

Seguridad Social y corresponde a la licitación pública 1607 - 13 - LP08.

Ningún conflicto de intereses por declarar.

Recibido el 9 de enero de 2012, aceptado el 23 de mayo de 2012.

Correspondencia a:

Dr. Rubén Alvarado

Escuela de Salud Pública. Facultad de Medicina. Universidad de Chile. Independencia 938, Santiago.

Fono: (56-2) 9786150. E-mail: ralvarado@med. uchile.cl

\section{Validación de un cuestionario para evaluar riesgos psicosociales en el ambiente laboral en Chile}

\author{
RUBÉN ALVARADO ${ }^{1}$, JUAN PÉREZ-FRANCO ${ }^{2,3}$, \\ NADIA SAAVEDRA ${ }^{3}$, CLAUDIO FUENTEALBA ${ }^{2,3}$, ALEX ALARCÓN $^{1}$, \\ NELLA MARCHETTI $^{1}$, WALDO ARANDA ${ }^{1}$
}

\section{Validation of a questionnaire for psychosocial risk assessment in the workplace in Chile}

Background: The measurement of psychosocial risk among workers is becoming increasingly important. Aim: To adapt, validate and standardize a questionnaire to measure psychosocial risks in the workplace. Material and Methods: The Spanish version of the Copenhagen Psychosocial Questionnaire was adapted and evaluated. Its contents were first validated with a panel of experts. Afterwards a semantic adaptation of the questionnaires was carried out applying it to a pilot sample. Finally, it was applied to 1,557 workers (65\% men). Results: A preliminary questionnaire containing 97 questions was constructed. A good item-test correlation was found, the factorial structure was similar to the original questionnaire and it had a good internal consistency, convergent validity with the Goldberg Health Questionnaire and test-retest correlation. Ranges for the different dimensions and sub-dimensions of psychosocial risk were calculated by tertiles. Conclusions: The resulting questionnaire is useful for measuring psychosocial risk factors at work, with good psychometric properties.

(Rev Med Chile 2012; 140: 1154-1163).

Key words: Occupational Health; Psychosocial factors; Questionnaires.
L as condiciones laborales en el mundo han experimentado grandes cambios en las últimas décadas, trayendo mayores exigencias para los trabajadores y menor capacidad de control sobre sus tareas ${ }^{1-4}$, lo que ha sido identificado como fuentes de estrés laboral y asociado al desarrollo de diversas patologías: osteomuscular ${ }^{5}$, cardiovascular $^{6,7}$, mental ${ }^{8-12}$, al igual que al ausentismo ${ }^{13,14}$.

Si bien en Chile se han hecho varios esfuerzos para medir el estrés relacionado con el trabajo ${ }^{15-26}$, no existía en el país una metodología validada para medir estos riesgos psicosociales del ambiente laboral.

Para esto se requiere un instrumento de medición de riesgos psicosociales que recoja información desde los trabajadores, construido con un riguroso diseño metodológico ${ }^{27}$. El Copenhagen Psychosocial Questionnaire (COPSOQ) ${ }^{28}$, del Ins- tituto Nacional de Seguridad y Salud Laboral de Dinamarca, fue traducido, validado y estandarizado en castellano ${ }^{29-31}$ y denominado Cuestionario ISTAS 21. El COPSOQ incluyó dimensiones de influyentes modelos de riesgo psicosocial ocupacional, pero también incluye otras dimensiones que permiten una comprensión más amplia de las condiciones del trabajo ${ }^{32}$. Alvarado et al adaptaron y validaron en Chile la versión media del cuestionario ISTAS 21 para población trabajadora $^{33}$. Posteriormente, la Superintendencia de Seguridad Social decidió validar a nivel nacional la versión en castellano del COPSOQ (ISTAS 21), con la colaboración de la Dirección del Trabajo, el Instituto de Salud Pública de Chile y la Escuela de Salud Pública de la Universidad de Chile, con la asesoría directa de los autores del ISTAS 21.

Este trabajo presenta los resultados de la vali- 
Validación de cuestionario psicosocial ISTAS 21 - R. Alvarado et al

dación y estandarización del cuestionario ISTAS 21 (versión extendida) para la población de trabajadores de Chile.

\section{Material y Método}

El proceso de validación y estandarización del instrumento se realizó en cuatro etapas: 1) validación de contenido; 2) adaptación semántica; 3) validación de constructo, de criterio y fiabilidad; y 4) estandarización a la población chilena.

\section{Descripción del instrumento ISTAS 21}

Se utilizó la versión larga del Cuestionario ISTAS 21, provista directamente por sus autores ${ }^{29}$. Este instrumento evalúa 5 dimensiones, que a su vez contienen varias sub-dimensiones (Tabla 1 ).

Cada pregunta tiene 5 opciones, con una puntuación de 0 a 4 . El mayor puntaje indica un mayor riesgo. Los puntajes se expresan como porcentaje del máximo valor que es posible alcanzar.

\section{Validación de contenido}

Se realizó un taller con 26 expertos de diversas instituciones públicas, privadas, académicas y sindicales, relacionadas con el tema. Los autores del instrumento ISTAS 21 participaron a través de teleconferencia. El grupo de expertos decidió mantener las mismas dimensiones y sub-dimensiones, agregó 7 preguntas (en 6 sub-dimensiones) y modificó el lenguaje en otras 28 preguntas (dentro de 14 sub-dimensiones), dando origen a una versión preliminar del cuestionario.

\section{Adaptación semántica}

Para esta etapa, la versión preliminar fue aplicada a una muestra intencionada de 50 personas, hombres y mujeres, que representaban todas las categorías ocupacionales planteadas por la OIT (CIUO-08, Clasificación Internacional Uniforme de Ocupaciones). Se realizaron modificaciones en 25 preguntas, dentro de 14 sub-dimensiones.

\section{Validación de constructo, de criterio y fiabilidad}

Se diseñó una muestra representativa de los trabajadores del país, estratificada en base a las ramas de actividad económica que son consideradas por el INE (10 grupos) y por sexo. Se utilizó una estrategia de muestreo polietápica, en cinco ciudades del país (Antofagasta, Coquimbo, Santiago,

Tabla 1. Dimensiones y subdimensiones de riesgo psicosocial contenidas en el instrumento SUSESO-ISTAS21

\begin{tabular}{|ll|}
\hline Dimensiones & Sub-dimensiones \\
\hline Exigencias psicológicas & Exigencias cuantitativas \\
& Exigencias cognitivas \\
& Exigencias emocionales \\
& Exigencias de esconder emociones \\
& Exigencias sensoriales \\
\hline Trabajo activo y desarrollo de habilidades & Influencia en el trabajo \\
& Posibilidades de desarrollo \\
& Control sobre el tiempo de trabajo \\
& Sentido del trabajo \\
& Integración en la empresa \\
\hline Apoyo social en la empresa y calidad del liderazgo & Claridad de rol \\
& Conflicto de rol \\
& Calidad de liderazgo \\
& Calidad de la relación con compañeros \\
& Calidad de la relación con sus superiores \\
& Posibilidades de relación social \\
\hline Compensaciones & Estima \\
Inseguridad respecto a las condiciones del contrato \\
Inseguridad respecto a las características del trabajo que realiza \\
\hline Doble presencia
\end{tabular}


Rancagua y Concepción). Se escogió una muestra de hogares; usando los mapas de las ciudades, se seleccionaron al azar las manzanas y luego las casas. Dentro de cada casa, se usó una tabla de Kish para seleccionar una persona. Cada encuestador debía completar una cuota de entrevistas por sexo y por rama de actividad económica.

La muestra final fue de 1.557 trabajadores, con 535 mujeres $(34,4 \%)$. La distribución por sexo y rubro de actividad económica se presenta en la Tabla 2. Las edades fluctuaron entre los 18 y 80 años, con un promedio de $37,0 \pm 12,1$ años. El promedio para el número de años de estudio fue de 14,4 $\pm 3,5$, con una mediana en 15 años.

Análisis de la heterogeneidad de los items. Los 97 ítems de la versión preliminar del instrumento resultaron ser heterogéneos. Ninguno mostró más de $80 \%$ de las respuestas en una sola de las opciones.

Correlación ítem-test. El análisis de correlación (prueba de Spearman) muestra que cada ítem está asociado positiva y significativamente $(\mathrm{p}<0,05)$ con la sub-dimensión a la que pertenece y con la dimensión respectiva. De igual forma, todas las sub-dimensiones se asociaron en forma positiva $y$ de manera significativa $(\mathrm{p}<0,05)$ con sus respectivas dimensiones.
Análisis factorial. Se utilizó el método de análisis factorial de componentes principales, con una solución final basada en una rotación Equamax. De esta forma se minimizó tanto el número de variables que saturan alto en un factor, como el número de factores necesarios para explicar una variable. Se excluyeron los factores que no superen el criterio de la raíz latente y no se consideraron en la interpretación de contenidos las saturaciones inferiores a 0,30. La pertinencia de realizar un análisis factorial se evaluó con el índice de adecuación muestral de Kaiser-Meyer-Oklin (KMO) ${ }^{34}$. En la Tabla 3 se muestra el resultado de este análisis factorial.

Para la dimensión de "exigencias psicológicas", se encontró una solución factorial que contenía 5 factores $(\mathrm{KMO}=0,869)$ que explican el $58,31 \%$ de la varianza. Todos los factores encontrados se correspondían exactamente con las sub-dimensiones de la dimensión de "exigencias psicológicas". En cuanto a los reactivos específicos, todos saturaban $(>0,30)$ en los factores que correspondían a las sub-dimensiones a las cuales pertenecían y todos presentaron una consistencia interna adecuada.

$\mathrm{El}$ análisis factorial de la dimensión "trabajo activo y desarrollo de habilidades" (KMO = 0,891 ) extrajo 6 factores que explican $55,6 \%$ de la

Tabla 2. Distribución de la muestra por sexo, edad y número de años de estudio según rubro de actividad económica

\begin{tabular}{|c|c|c|c|c|c|c|c|}
\hline \multirow[b]{3}{*}{ Rubro de actividad económica } & \multicolumn{4}{|c|}{ Sexo } & \multicolumn{2}{|c|}{$\begin{array}{c}\text { n de años de } \\
\text { estudio }\end{array}$} & \multirow{3}{*}{$\begin{array}{l}\text { Edad } \\
\text { Pro }\end{array}$} \\
\hline & \multicolumn{2}{|c|}{ Hombre } & \multicolumn{2}{|c|}{ Mujer } & \multirow{2}{*}{$\begin{array}{c}\text { H } \\
\text { Pro }\end{array}$} & \multirow{2}{*}{$\begin{array}{c}\text { M } \\
\text { Pro }\end{array}$} & \\
\hline & $\mathbf{n}$ & $\%$ & $\mathbf{n}$ & $\%$ & & & \\
\hline Transporte, almacenamiento y comunicaciones & 91 & 80,5 & 22 & 19,5 & 14,1 & 14,0 & 36,6 \\
\hline Servicios comunales, sociales y personales & 94 & 40,5 & 138 & 59,5 & 16,3 & 17,0 & 39,7 \\
\hline Industria manufacturera & 129 & 84,3 & 24 & 15,7 & 14,0 & 13,0 & 38,6 \\
\hline Explotación de minas y canteras & 57 & 95,0 & 3 & 5,0 & 14,6 & 5,0 & 40,5 \\
\hline $\begin{array}{l}\text { Establecimientos financieros, seguros, bienes inmuebles y } \\
\text { servicios de préstamos }\end{array}$ & 67 & 56,8 & 51 & 43,2 & 15,5 & 16,0 & 36,7 \\
\hline Electricidad, gas y agua & 36 & 94,7 & 2 & 5,3 & 13,6 & 12,5 & 35,2 \\
\hline Construcción & 101 & 90,2 & 11 & 9,8 & 13,7 & 13,5 & 37,3 \\
\hline Comercio por mayor o menor, restaurantes y hoteles & 115 & 57,2 & 86 & 42,8 & 13,8 & 13,0 & 33,9 \\
\hline Agricultura, caza, silvicultura y pesca & 92 & 71,9 & 36 & 28,1 & 12,4 & 12,0 & 37,4 \\
\hline No especificado & 240 & 59,7 & 162 & 40,3 & 14,6 & 15,0 & 36,0 \\
\hline Total & 1.022 & 65,6 & 535 & 34,4 & & & \\
\hline
\end{tabular}


Validación de cuestionario psicosocial ISTAS 21 - R. Alvarado et al

Tabla 3. Resultado del análisis factorial aplicado a cada una de las dimensiones

\begin{tabular}{|c|c|c|c|c|}
\hline Factor & $\begin{array}{c}\% \text { Var. } \\
\text { explicada }\end{array}$ & $\begin{array}{c}\% \text { Var. } \\
\text { acumulada }\end{array}$ & & KMO \\
\hline & & & Exigencias psicológicas & 0,86 \\
\hline 1 & 15,18 & 15,18 & Exigencias cognitivas & \\
\hline 2 & 13,51 & 28,69 & Exigencias cuantitativas & \\
\hline 3 & 13,45 & 42,14 & Exigencias sensoriales & \\
\hline 4 & 8,53 & 50,67 & Exigencias emocionales & \\
\hline \multirow[t]{2}{*}{5} & 7,64 & 58,31 & Exigencias de esconder emociones & \\
\hline & & & Trabajo activo y desarrollo de habilidades & 0,89 \\
\hline 1 & 10,71 & 10,71 & Integración en la empresa & \\
\hline 2 & 10,48 & 21,19 & Sentido del trabajo & \\
\hline 3 & 10,26 & 31,45 & Posibilidades de desarrollo & \\
\hline 4 & 9,28 & 40,72 & Control sobre el tiempo de trabajo & \\
\hline 5 & 8,23 & 48,95 & Influencia sobre las condiciones generales de trabajo & \\
\hline \multirow[t]{2}{*}{6} & 6,34 & 55,29 & Influencia sobre las condiciones específicas de trabajo & \\
\hline & & & Apoyo social en la empresa y calidad del liderazgo & 0,92 \\
\hline 1 & 15,23 & 15,23 & Calidad de liderazgo & \\
\hline 2 & 12,93 & 28,16 & Calidad de la relación con compañeros & \\
\hline 3 & 11,38 & 39,54 & Calidad de la relación con sus superiores & \\
\hline 4 & 11,06 & 50,60 & Conflicto de rol & \\
\hline 5 & 10,09 & 60,69 & Claridad de rol & \\
\hline \multirow[t]{2}{*}{6} & 6,38 & 67,07 & Posibilidades de relación social & \\
\hline & & & Compensaciones & 0,84 \\
\hline 1 & 21,28 & 21,28 & Estima & \\
\hline 2 & 19,94 & 41,22 & Inseguridad respecto a las condiciones del contrato & \\
\hline \multirow[t]{2}{*}{3} & 18,87 & 60,09 & Inseguridad respecto a las condiciones del trabajo que realiza & \\
\hline & & & Doble presencia & 0,60 \\
\hline 1 & 38,13 & 38,13 & Preocupación por tareas domésticas & \\
\hline 2 & 37,22 & 75,35 & Carga de tareas domésticas & \\
\hline
\end{tabular}

varianza total. Los primeros 4 factores correspondían exactamente con las sub-dimensiones que constituyen la dimensión mayor. La única subdimensión cuyos ítems saturaron en dos factores distintos fue "influencia". Más precisamente, se observó que 4 ítems se referían a las condiciones generales de trabajo y saturaban fuertemente $(>0,60)$ sobre el factor 5 , y que 3 ítems que se referían a la calidad, horario y orden del trabajo saturaban $(>0,40)$ en el factor 6 . Pero, al estudiar la consistencia interna se encontró que esta era baja, por lo cual se tomó la decisión de conservar la sub-dimensión completa con sus ítems originales.

En cuanto a la dimensión "apoyo social en la empresa y calidad de liderazgo", el análisis factorial obtuvo 6 factores $(67,07 \%$ de la varianza total explicada), de los cuales 4 correspondían exactamente con las sub-dimensiones "claridad de rol" (factor 5), "conflicto de rol” (factor 4), "calidad de liderazgo" (factor 1) y "posibilidades de relación social” (factor 6). El factor 2 reunía 6 ítems: 3 correspondiente a las sub-dimensión de "previsibilidad" y 3 a la de "apoyo social de superiores". En cuanto al factor 3, reunía 5 ítems que pertenecían originalmente a las sub-dimensiones de "sentido de grupo" y "apoyo social de compañeros". Esto llevó a crear dos nuevas sub-dimensiones: "calidad de la relación con superiores" (factor 3 ) y "calidad de la relación con sus compañeros" (factor 2). El análisis de consistencia hecho para estas dos nuevas sub-dimensiones mostró índices más que satisfactorios (Tabla 4). 
Tabla 4. Consistencia interna para las dimensiones y sub-dimensiones psicosociales de riesgo laboral (Alfa de Cronbach)

\begin{tabular}{|c|c|c|c|}
\hline Dimensiones & Sub-dimensiones & $\alpha$ & $\begin{array}{l}\mathrm{n}^{\circ} \text { de } \\
\text { ítems }\end{array}$ \\
\hline \multirow[t]{6}{*}{ Exigencias psicológicas } & & 0,857 & 23 \\
\hline & Exigencias cuantitativas & 0,793 & 7 \\
\hline & Exigencias cognitivas & 0,844 & 8 \\
\hline & Exigencias emocionales & 0,850 & 2 \\
\hline & Exigencias de esconder emociones & 0,766 & 2 \\
\hline & Exigencias sensoriales & 0,818 & 4 \\
\hline \multirow[t]{6}{*}{$\begin{array}{l}\text { Trabajo activo y desarrollo de } \\
\text { habilidades }\end{array}$} & & 0,859 & 25 \\
\hline & Influencia en el trabajo & 0,688 & 7 \\
\hline & Posibilidades de desarrollo & 0,784 & 7 \\
\hline & Control sobre el tiempo de trabajo & 0,684 & 4 \\
\hline & Sentido del trabajo & 0,773 & 3 \\
\hline & Integración en la empresa & 0,819 & 4 \\
\hline \multirow[t]{7}{*}{$\begin{array}{l}\text { Apoyo social en la empresa y } \\
\text { calidad del liderazgo }\end{array}$} & & 0,915 & 26 \\
\hline & Claridad de rol & 0,710 & 4 \\
\hline & Conflicto de rol & 0,775 & 5 \\
\hline & Calidad de liderazgo & 0,914 & 6 \\
\hline & Calidad de la relación con compañeros & 0,844 & 6 \\
\hline & Calidad de la relación con sus superiores & 0,816 & 5 \\
\hline & Posibilidades de relación social & 0,578 & 3 \\
\hline \multirow[t]{4}{*}{ Compensaciones } & & 0,829 & 13 \\
\hline & Estima & 0,794 & 5 \\
\hline & Inseguridad respecto a las condiciones del contrato & 0,803 & 5 \\
\hline & Inseguridad respecto a las características del trabajo que realiza & 0,803 & 3 \\
\hline \multirow[t]{3}{*}{ Doble presencia } & & 0,644 & 4 \\
\hline & Carga de tareas domésticas & 0,660 & 2 \\
\hline & Preocupación por tareas domésticas & 0,662 & 2 \\
\hline
\end{tabular}

La dimensión "compensaciones” también presentó una solución de 3 factores $(\mathrm{KMO}=0,840)$ que explicaban 60,09\% de varianza. Los ítems que saturaban $(>0,7)$ en el primer factor se correspondían precisamente con la sub-dimensión "estima", por lo que dicha sub-dimensión se mantuvo igual que en la versión original. Por otro lado, los ítems que inicialmente pertenecían a la sub-dimensión de "inseguridad laboral" presentaron saturaciones en dos factores distintos, los que después del análisis conceptual fueron definidos como "inseguridad respecto a las condiciones generales del contrato" (factor 2) e "inseguridad respecto a las características específicas del trabajo que realiza" (factor 3). La consistencia interna de estas dos nuevas sub-dimensiones resultó muy buena, con un $\alpha=0,803$ en ambas (Tabla 4).

Finalmente, del análisis factorial de la dimensión "doble presencia" se extrajeron dos factores que explican $75,36 \%$ de varianza. Los cuatro ítems de esta dimensión quedaron separados: dos ítems saturaron $(>0,80)$ en el primer factor que mide la "cantidad de carga en tareas domésticas" (factor 2) y los dos ítems restantes tenían cargas factoriales elevadas $(>0,80)$ sobre un factor que denominamos "preocupación por tareas domésticas" (factor 1). La consistencia interna también fue satisfactoria (Tabla 4).

Análisis de consistencia interna. Se utilizó el índice Alfa de Cronbach $(\alpha)$ para valorar la con- 
Validación de cuestionario psicosocial ISTAS 21 - R. Alvarado et al

Tabla 5. Correlación de Spearman entre las Dimensiones y Sub-dimensiones con el puntaje en el GHQ-12

\begin{tabular}{|c|c|c|}
\hline & GHQ-12 & $\mathbf{p}$ \\
\hline Exigencias psicológicas & $0,290 * *$ & $<0,001$ \\
\hline Exigencias psicológicas cuantitativas & $0,257 * *$ & $<0,001$ \\
\hline Exigencias psicológicas cognitivas & $0,050^{*}$ & 0,024 \\
\hline Exigencias psicológicas emocionales & $0,303^{* *}$ & $<0,001$ \\
\hline Exigencias psicológicas de esconder emociones & $0,222 * *$ & $<0,001$ \\
\hline Exigencias psicológicas sensoriales & 0,022 & 0,195 \\
\hline Trabajo activo y posibilidades de desarrollo & $0,253^{* *}$ & $<0,001$ \\
\hline Influencia & $0,162^{* *}$ & $<0,001$ \\
\hline Control sobre el tiempo de trabajo & $0,189 * *$ & $<0,001$ \\
\hline Posibilidades de desarrollo en el trabajo & $0,124 * *$ & $<0,001$ \\
\hline Sentido del trabajo & $0,155^{* *}$ & $<0,001$ \\
\hline Integración en la empresa & $0,221 * *$ & $<0,001$ \\
\hline Compensaciones & $0,261 * *$ & $<0,001$ \\
\hline Inseguridad respecto al contrato de trabajo & $0,164^{* *}$ & $<0,001$ \\
\hline Inseguridad respecto a las características de trabajo & $0,161 * *$ & $<0,001$ \\
\hline Estima & $0,312 * *$ & $<0,001$ \\
\hline Apoyo social en la empresa y calidad de liderazgo & $0,312^{* *}$ & $<0,001$ \\
\hline Claridad de rol & $0,189 * *$ & $<0,001$ \\
\hline Conflicto de rol & $0,249 * *$ & $<0,001$ \\
\hline Calidad de liderazgo & $0,261 * *$ & $<0,001$ \\
\hline Calidad de la relación con superiores & $0,225^{* *}$ & $<0,001$ \\
\hline Calidad de la relación con compañeros de trabajo & $0,204^{* *}$ & $<0,001$ \\
\hline Doble presencia & $0,195^{* *}$ & $<0,001$ \\
\hline Preocupación por tareas domésticas & $0,110^{* *}$ & $<0,001$ \\
\hline Carga de tareas domésticas & $0,203^{* *}$ & $<0,001$ \\
\hline
\end{tabular}

${ }^{*}$ Correlación de Spearman significativa a un nivel $<0,05$ (test unilateral). ${ }^{* *}$ Correlación de Spearman significativa a un nivel $<0,001$ (test unilateral).

sistencia interna y los resultados se presentan en la Tabla 4.

La dimensión de "exigencias psicológicas" muestra un buen nivel de consistencia interna $(\alpha=0,857)$. Los resultados para cada una de sus sub-dimensiones también mostraron buenos índices de consistencia interna, con valores que fluctuaron entre 0,766 y 0,850 .

La dimensión de "trabajo activo y desarrollo de habilidades" también obtuvo un muy buen nivel de consistencia interna con un alfa de 0,859. Los resultados para sus sub-dimensiones estuvieron entre 0,684 y 0,819 .

La dimensión "apoyo social en el trabajo y calidad del liderazgo" obtuvo un alfa de 0,915.
Dentro de esta dimensión se encontraba la subdimensión "posibilidades de relación" que obtuvo un alfa de 0,578 , razón por la cual se procedió a eliminarlo (incluía 3 preguntas). El resto de las cinco sub-dimensiones mostraron indicadores más que satisfactorios, con valores que fluctuaron entre 0,710 y 0,914 .

La dimensión de "compensaciones" también mostró un buen indicador de consistencia interna $(\alpha=0,829)$. Las tres sub-dimensiones presentaron buenos indicadores, oscilando entre 0,794 y 0,803 .

La dimensión de doble presencia fue la que obtuvo el alfa más bajo (con un valor de 0,644 ), pero aún dentro de límites aceptables. Sus dos 
sub-dimensiones fluctuaron alrededor de esta cifra $(0,660$ y 0,662$)$.

Análisis de validez convergente. Se estudió a través de un análisis de correlación con la prueba de Spearman entre los puntajes totales obtenidos en cada dimensión y sub-dimensión, con el puntaje en el Goldberg Health Questionnaire en su versión de 12 preguntas (GHQ-12) ${ }^{35}$. Este último es un cuestionario ampliamente utilizado para medir el nivel de malestar psicológico. En la Tabla 5 se muestran los resultados de esta correlación. Las 5 grandes dimensiones presentan una correlación positiva y estadísticamente significativa con el puntaje obtenido en el GHQ-12, lo que indicaría que a mayor puntaje en las dimensiones de este instrumento, habría mayor malestar psicológico. La asociación más fuerte se presenta en la dimensión "apoyo social en el trabajo y calidad del liderazgo" $(r=0,312, p<0,001)$, y la más débil en "doble presencia" $(\mathrm{r}=0,195, \mathrm{p}<0,001)$.

En el caso de las sub-dimensiones, casi todas presentaron una asociación positiva y estadísticamente significativa (a nivel de $\mathrm{p}<0,001$ ) con el puntaje del GHQ-12. La sub-dimensión "exigencias psicológicas cognitivas" mostró una asociación lineal menos marcada $(\mathrm{r}=0,05, \mathrm{p}=$ $0,024)$ pero igualmente significativa. La única sub-dimensión que no presentó una asociación estadísticamente significativa fue "exigencias psicológicas sensoriales” ( $\mathrm{r}=0,022, \mathrm{p}=0,195)$.

Análisis de la fiabilidad test-retest. Se realizó a través de una prueba de correlación entre la primera aplicación del cuestionario y una segunda aplicación (que se hizo a una sub-muestra de 157 personas, con igual distribución por sexo y rubro económico de la muestra original). Todas las correlaciones entre el test y el re-test fueron elevadas (con valores de $r$ entre 0,661 y 0,730 ), positivas y estadísticamente significativas (todas las dimensiones y sub-dimensiones obtuvieron un $\mathrm{p}<0,001)$. Esto indica que el instrumento tiene una buena estabilidad y fiabilidad temporal.

\section{Estandarización a la población chilena}

Para el cálculo de los puntajes se siguió el procedimiento establecido en el Manual ISTAS 21(29). Los valores obtenidos son expresados como porcentaje respecto del puntaje máximo posible que se puede obtener en cada sub-dimensión. Y para el caso de las dimensiones, los puntajes corresponden a un promedio de las sub-dimensiones que esta contiene. A continuación, se procedió a estimar los valores que permiten separar los puntajes por terciles (tal como se realizó en España). Con esto se busca establecer los rangos "bajo", "medio" y "alto" de exposición a cada uno de los factores de riesgo psicosocial evaluados por este instrumento (Tabla 6).

\section{Discusión}

El cuestionario COPSOQ, en su versión española ISTAS 21, fue sometido a un proceso de adaptación, validación y estandarización en una muestra de 1.557 personas, representativa de la población trabajadora chilena. El propósito del instrumento es detectar y medir los riesgos psicosociales en el trabajo. Su aplicación en el medio laboral debiera permitir la detección y mitigación de las condiciones de riesgo psicosocial de los trabajadores.

El amplio espectro de aspectos psicosociales que abarca el instrumento, así como el consenso entre expertos con que se inició el proceso, sugieren una alta validez de contenido.

El análisis de validez de constructo mostró que las cinco dimensiones globales definidas teóricamente ("exigencias psicológicas", "trabajo activo y posibilidades de desarrollo", "apoyo social en la empresa y calidad del liderazgo", "compensaciones" y "doble presencia") pudieron ser confirmadas por análisis factorial.

La gran mayoría de las escalas obtuvo un alfa de Cronbach mayor que el nivel convencional de 0,700 , y la mitad obtuvo un alfa mayor a 0,800 , señalando su buena consistencia interna. Por otro lado, en el análisis test-retest todas las correlaciones fueron estadísticamente significativas, lo que muestra una alta estabilidad temporal.

Las nuevas dimensiones "calidad de la relación con supervisores" y "calidad de la relación con compañeros" que surgieron durante este proceso de validación, son similares a las dimensiones "social support from supervisors" y "social support from colleagues" de la versión más reciente del COPSOQ ${ }^{36}$. De igual forma, el COPSOQ II eliminó la dimensión "exigencias psicológicas sensoriales", que en nuestro estudio fue la que obtuvo la menor correlación con el GHQ-12. Ambos resultados permiten afirmar que la versión chilena de este cuestionario es consistente con la 
Validación de cuestionario psicosocial ISTAS 21 - R. Alvarado et al

Tabla 6. Rangos de puntajes por terciles para cada dimensión y sub-dimensión

\begin{tabular}{|c|c|c|c|}
\hline & “bajo" & "medio" & "alto" \\
\hline "Exigencias psicológicas" & $0-46,33$ & $46,34-59,64$ & $59,65-100$ \\
\hline Exigencias psicológicas cuantitativas & $0-28,56$ & $28,57-42,85$ & $42,86-100$ \\
\hline Exigencias psicológicas cognitivas & $0-59,37$ & $59,38-78,12$ & $78,13-100$ \\
\hline Exigencias psicológicas emocionales & $0-24,99$ & $25,00-49,99$ & $50,00-100$ \\
\hline Exigencias psicológicas de esconder emociones & $0-12,50$ & $12,51-49,99$ & $50,00-100$ \\
\hline Exigencias psicológicas sensoriales & $0-74,99$ & $75,00-93,74$ & $93,75-100$ \\
\hline “Trabajo activo y posibilidades de desarrollo” & $0-28,09$ & $28,10-42,14$ & $42,15-100$ \\
\hline Influencia & $0-39,28$ & $39,29-57,13$ & $57,12-100$ \\
\hline Control sobre el tiempo de trabajo & $0-24,99$ & $25,00-50,00$ & $50,01-100$ \\
\hline Posibilidades de desarrollo en el trabajo & $0-17,84$ & $17,85-35,70$ & $35,69-100$ \\
\hline Sentido del trabajo & 0 & $0,01-16,66$ & $16,67-100$ \\
\hline Integración en la empresa & $0-24,99$ & $25,00-56,24$ & $56,25-100$ \\
\hline "Apoyo social en la empresa y calidad de liderazgo" & $0-19,25$ & $19,26-32,58$ & $32,59-100$ \\
\hline Claridad de rol & 0 & $0,01-18,74$ & $18,75-100$ \\
\hline Conflicto de rol & $0-14,99$ & $15,00-35,00$ & $35,01-100$ \\
\hline Calidad de liderazgo & $0-20,82$ & $20,83-41,67$ & $41,68-100$ \\
\hline Calidad de la relación con superiores & $0-19,99$ & $20,00-35,00$ & $35,01-100$ \\
\hline Calidad de la relación con compañeros de trabajo & $0-12,49$ & $12,50-29,77$ & $29,78-100$ \\
\hline "Compensaciones" & $0-21,66$ & $21,67-42,78$ & $42,79-100$ \\
\hline Estima & $0-19,99$ & $20,00-35,00$ & $35,01-100$ \\
\hline Inseguridad respecto al contrato de trabajo & $0-20,00$ & $20,01-49,99$ & $50,00-100$ \\
\hline Inseguridad respecto a las características del trabajo & $0-8,32$ & $8,33-41,66$ & $41,67-100$ \\
\hline "Doble presencia" & $0-18,75$ & $18,76-37,50$ & $37,51-100$ \\
\hline Preocupación por tareas domésticas & $0-12,50$ & $12,51-37,50$ & $37,51-100$ \\
\hline Carga de tareas domésticas & $0-12,50$ & $12,51-37,50$ & $37,51-100$ \\
\hline
\end{tabular}

experiencia acumulada a la fecha en esta área de trabajo.

Debe destacarse la rigurosa metodología de selección de una muestra estratificada por actividad económica representativa de la población trabajadora chilena, que lo hace diferenciarse del original danés que se hizo con una muestra de población general estratificada por edad, que luego se ordenó por actividad económica ${ }^{28}$, y de la adaptación alemana que se hizo con los cuestionarios que retornaron por correo al equipo investigador, con lo que hubo una sobrerrepresentación de población de alto nivel socio-cultural ${ }^{37}$. La versión española, en cambio, se realizó inicialmente con población trabajadora de Navarra ${ }^{29}$ y luego se extendió a una muestra representativa de la población trabajadora de toda España ${ }^{31}$.

Creemos así que se pone a disposición de los trabajadores, empleadores, técnicos vinculados a la salud ocupacional e investigadores chilenos, un instrumento que permite diagnosticar y medir el nivel de riesgo psicosocial en el trabajo. Este instrumento debiera permitir desarrollar programas de prevención y de intervención en el trabajo que mejoren o eliminen los riesgos detectados, y contribuir de esta manera a una mejor calidad de vida de los trabajadores chilenos. Que este objetivo se logre sólo puede decirlo el tiempo y el uso sistemático que se pueda hacer de él en los distintos ámbitos de aplicación.

\section{Referencias}

1. Oficina Internacional del Trabajo. Cambios en el mundo del trabajo, Memoria del Director General, Ginebra, 2006.

2. Oficina Internacional del Trabajo. Evolución de los salarios en América Latina 1995-2006. Santiago, 2008. 
3. Bahamondes Pavez C, Wilde B, Hinrichs S, Schüpath H. Cambios en la Organización del Trabajo. Dirección Orientada a los Resultados y sus Implicaciones para los Empleados. Cienc Trab 2009; 32: 102-10.

4. Superintendencia de Seguridad Social. Departamento Actuarial. Aumento del gasto en subsidios por incapacidad laboral. Evolución de las licencias médicas curativas. 2008.

5. Ariëns GAM, Bongers PM, Hoogendoorn WE, Houtman ILD, Wal G. van der, Mechelen W. van. High quantitative job demands and low co-worker support are risk factors for neck pain: results of a prospective cohort study. Spine 2001; 26 (17): 1896-903.

6. Belkic K, Landsbergis PA, Schnall PL, Baker D. Is job strain a major source of cardiovascular risk? Scand J Work Environ Health 2004; 30 (2): 85-128.

7. Kivimäki M, Leino-Arjas P, Luukkonen R, Riihimäki H, Vahtera J y Kirjonen J. Work stress and risk of coronary mortality: prospective cohort study of industrial employees. BMJ 2002; 325: 857-63.

8. Kawakami N, Araki S, Kawashima M. Effects of job stress on occurrence of major depression in Japanese industry: a case-control study nested in a cohort study. J Occup Med 1990; 32 (8): 722-5.

9. Niedhammer I, Goldberg M, Leclerc A, Bugel I, David S. Psychosocial factors at work and subsequent depressive symptomsin the Gazel cohort. Scand J Work Environ Health 1998; 24 (3): 197-205.

10. Thierriault P, Streit U, Rhéaume J. Situation paradoxale dans l'organisation du travail: une menace pour la santé mentale des travailleurs. Santé mentale au Quebéc 2004; 29 (1): 173-200.

11. Rugulies R, Bultmann U, Aust B, Burr H. Psychosocial work environment and incidence of severe depressive symptoms: prospective findings from a 5-year followup of the Danish work environment cohort study. Am J Epidemiol 2006; 163 (10): 877-87.

12. Ansoleaga E, Toro JP. Factores psicosociales laborales asociados a riesgo de sintomatología depresiva en trabajadores de una empresa minera. Salu trab (Maracay) 2010; 18 (1): 7-16.

13. North FM, Leonard S, Feeney A, Shipley M, Marmot M. Psychosocial Work Environment and Sickness Absence among British Civil Servants: The Whitehall II Study. Am J Public Health 1996; 86: 332-40.

14. Ala-Mursula L, Vahtera J, Kivimäki M, Kevin MV, Pentti J. Employee control over working times: associations with subjective health and sickness absences. J Epidemiol Community Health 2002; 56: 272-8.

15. Trucco M, Campusano ME, Larraín S. Un cuestionario para detectar desórdenes emocionales: estudio de valida- ción preliminar. Rev Chil Neuro-Psiquiat 1979; 7: 20-5.

16. Trucco M, Valenzuela P. Una batería de cuestionarios para el estudio de estrés ocupacional. Rev Chil NeuroPsiquiat 1998; 36: 159-65.

17. Guic E, Bilbao MA, Bertin C. Estrés laboral y salud en una muestra de ejecutivos chilenos. Rev Med Chile 2002; 130 (10): 1101-12.

18. Vera A, Sepúlveda R, Contreras G. Auto-reporte de síntomas físicos y correlatos psicosociales en trabajadores de la minería. Cienc Trab 2006; 8( 20): 74-8.

19. Leiva H, León F, Medina C. Síndrome de Burnout en funcionarios de servicios pediátricos de la Sexta Región. Rev Chil Salud Pública 2004; 8 (3): 137-42.

20. Quintanilla M. Prevalencia del síndrome de Burnout en las enfermeras de la Unidad de Paciente Crítico del Hospital del Trabajador de Santiago y una propuesta de intervención. Rev Chil Med Intensiv 2004; 19 (1): 33-7.

21. Ordenes N. Prevalencia de Burnout en trabajadores del Hospital Roberto del Río. Rev Chil Pediatr 2004; 75 (5): 449-54.

22. Olivares V, Vera A, Juárez A. Prevalencia del síndrome de quemarse por el trabajo (Burnout) en una muestra de profesionales que trabajan con personas con discapacidades en Chile. Cienc Trab 2009; 11 (32): 63-71.

23. Vera A, Ravanal I, Cancino L, Carrasco C, Contreras G, Arteaga O. Síndrome de Burnout e inteligencia emocional: una análisis con enfoque psicosocial en una agencia estatal chilena. Cienc Trab 2007; 9 (24): 51-54.

24. Sandoval C, Sanhueza L, Borjas R, González P, Odgers C, Najafzadeh-Tabrizi S. Síndrome de Burnout en el personal profesional del Hospital de Yumbel. Cuad Méd Soc 2006; 46 (4): 268-73.

25. Manso-Pinto J. Estructura factorial del Maslach Burnout Inventory: version Human Services Survey en Chile. Interam J Psychol 2006; 40 (1): 111-4.

26. Guic E, Mora P, Rey R, Robles A. Estrés organizacional y salud en funcionarios de centros de atención primaria de una comuna de Santiago. Rev Med Chile 2006; 134: 447-55.

27. Kompier M. Assessing the psychosocial work environment - "subjective" versus "objective" measurement. Scand J Work Environ Health 2005; 31 (6): 405-8.

28. Kristensen TS, Hannerz H, Høgh A, Borg V. The Copenhagen Psychosocial Questionnaire-a tool for assessment and improvement of the psychosocial work environment. Scand J Work Environ Health 2005; 31 (6): 438-49.

29. Moncada S, Llorens C, Kristensen TS. Manual del Método ISTAS 21 COPSOQ, versión castellana del Cuestionario Psicosocial de Copenhagen. ISTAS. 2002. 
Validación de cuestionario psicosocial ISTAS 21 - R. Alvarado et al

30. Moncada S, Llorens C, Navarro A, Kristensen TS. ISTAS21: Versión en lengua castellana del cuestionario psicosocial de Copenhague (COPSOQ). Arch Prev Riesgos Labor 2005; 8: 18-29.

31. Moncada S, Llorens C, Font A, Galtés A, Navarro A. Exposición a riesgos psicosociales entre la población asalariada en España (2004-2005): Valores de referencia de las 21 dimensiones del cuestionario COPSOQ ISTAS 21. Rev Esp Salud Pública 2008; 82: 667-75.

32. Kristensen TS. A questionnaire is more than a questionnaire. Scand J Public Health 2010; 38 (Supp 3): 149-55.

33. Alvarado R, Marchetti N, Villalón M, Hirmas M, Pastorino MS. Adaptación y análisis psicométrico de un cuestionario para evaluar riesgos psicosociales en el trabajo en Chile: versión media del COPSOQ. Rev Chil
Salud Pública 2009; 13 (1): 7-16.

34. Thompson B. Exploratory and Confirmatory Factor Analysis. Understanding Concepts and Applications. Washington DC: American Psychological Association. 2004.

35. Goldberg D \& Wiliams P. Cuestionario de Salud General (GHQ). Guía para el usuario de las distintas versiones. España: Masson. 1996.

36. Pejtersen JH, Kristensen TS, Borg V y Bjorner JB. The second version of the Copenhagen Psychosocial Questionnaire. Scand J Public Health 2010; 38: 8-24.

37. Nübling M, Stößel U, Hasselhorn HM, Michaelis M, Hofmann F. Measuring psychological stress and strain at work: Evaluation of the COPSOQ Questionnaire in Germany. GMS Psychosoc Med 2006; 3: Doc05. 